\title{
Adaptive Tool Path Planning for 5-Axis Sculptured Surface Machining by Machining Strip Evaluation
}

\author{
Yuan-Shin Lee, Ph.D., P.E. (e-mail: yslee@eos.ncsu.edu; http://www.ie.ncsu.edu/yslee) \\ IE Dept., North Carolina State Univ., Raleigh, NC 27695-7906, U.S.A.
}

\begin{abstract}
This paper presents a new approach to non-isoparametric 5-axis NC tool path generation for complex surface machining by using the techniques of feasible machining strip evaluation. A searching algorithm is developed to find the parameter increments of adjacent cutter locations along orthogonal path intervals for non-isoparametric path generation. The techniques presented in this paper can be used to automate the non-isoparametric 5-axis cutter path generation and reduce the total machining time for $\mathrm{CAD} / \mathrm{CAM}$ systems.
\end{abstract}

\section{INTRODUCTION}

Tool paths for sculptured surface machining are traditionally generated by guiding cutters to trace along constant parametric curves on the surfaces [Deng 96, Lee 95a]. An example of the conventional constant parametric cutter path generation for sculptured surface machining is shown in Figure 1. The distance between the parallel trajectories is the CC path interval, which depends on the local surface shape, cutter size and the allowable scallop height remaining on the surface after machining [Chen 98, Jerard 91, Lin 96, Lee 96b]. To achieve machined surface quality, the scallops left on the part surface need to be controlled within allowable tolerance. In the constant parametric (isoparametric) path generation methods, tool path distribution is determined by calculating, at each path, the smallest tool path interval and using it as a constant interval for the next tool path [Altan 93, Ma 98]. One serious problem of the traditional (isoparametric) method is the inefficient machining due to the non-predictable scallop remaining on the part surface [Lin 96, Lee 97b, Suresh 98]. Since the next tool path is generated by the smallest path interval between adjacent tool paths, redundant machining overlap occurs between the two adjacent tool paths, which causes machining inefficiency. In this paper, we adopt a new approach for the determination of efficient tool paths by using the machining strip evaluation method and a non-isoparametric path generation algorithm, which results in shorter total tool path than the conventional constant parametric tool path methods achieve.

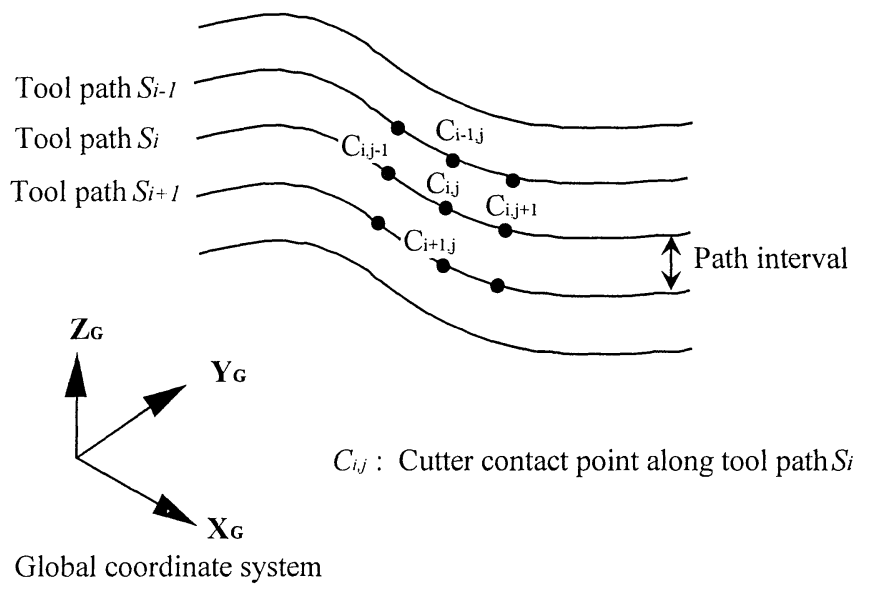

Figure 1. Constant parameter (Isoparametric) tool path distribution

The original version of this chapter was revised: The copyright line was incorrect. This has been corrected. The Erratum to this chapter is available at DOI: 10.1007/978-0-387-35392-0_40 


\section{FEASIBLE MACHINING STRIP EVALUATION}

Effective cutting shape analysis was developed in our earlier work presented in [Lee 96a, 97a, 98b]. During machining, a surface tolerance $h$ is given to maintain the machined surface accuracy. During machining, the machined region that lies within the required surface tolerance is called the machining strip (w) [Lee 97a], as shown in Figure 2. In Figure 2, the local surface curvature (normal to the cutting direction $\overrightarrow{\mathbf{X}_{\mathbf{L}}}$ ) is represented as $\kappa$. The radius of surface curvature can then be calculated as $\frac{1}{K}$. A local coordinate system is defined on the cutter contact point $\mathrm{C}^{*}$. The effective cutting edge $E(\theta)$ intersects the offset surface at points $C_{a}$ and $C_{b}$. The region between points $C_{a}$ and $C_{b}$ is the feasible machining strip, and the machined surface errors within the machining strip are within the given tolerance $h$, as shown in Figure 2. Details of finding the feasible machining strip $w$ can be found in our earlier work in [Lee 97a]. As shown in Figure 2, the machining strip width $w$ at the current cutting position $\mathrm{C}^{*}$ with tool orientation $\left(\lambda_{L}, \omega_{L}\right)$ can be found as:

$$
w=w_{a}+w_{b}
$$

It is noticed that, given the same tool orientation $\left(\lambda_{L}, \omega_{L}\right)$, the feasible machining strip width $w$ of the same effective cutting shape $E(\theta)$ in different surface regions has the relationship of $\left(w_{\text {concave }}>w_{\text {planar }}>w_{\text {convex }}\right)[$ Lee $97 \mathrm{a}]$.

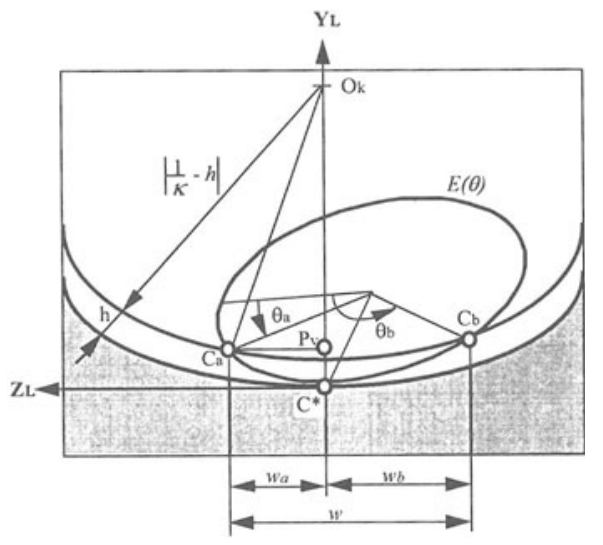

Cutting direction $(\mathbf{X L})$ out from the paper

Figure 2. Machining strip width $w$ at cutter contact point $C^{*} \quad$ [Lee 97a]

\section{TOOL PATH DISTRIBUTION BY MACHINING STRIP EVALUATION}

To generate cutter paths to achieve the required surface quality, the machining strips of the two adjacent cutter paths have to overlap each other to ensure no machined surface error exceeds the tolerance limit $h$. As shown in Figure 3, given a surface tolerance $h$, the machining strip width $w_{i+1}$ of the new adjacent tool path $S_{i+1}$ should be connected to the machining strip $w_{i}$ of the current tool path $S_{i}$ to ensure the required surface finish is satisfied. To ensure the scallop height is no larger than the required surface finish, a distance $D$ between the current cutter contact point $C_{i, j}$ and the adjacent cutter contact point $C_{i+1, j}$ should be no larger than the connected machining strips as follows [Lee 98a]: 


$$
w_{b, i}+w_{a, i+1} \geq D
$$

where, $w_{b, i}$ is the right portion of the machining strip $w_{i}$ at cutter contact point $C_{i, j}$, and $w_{a, i+1}$ is the left portion of the adjacent machining strip $w_{i+1}$ at $C_{i+1, j}$, as shown in Figure 3.

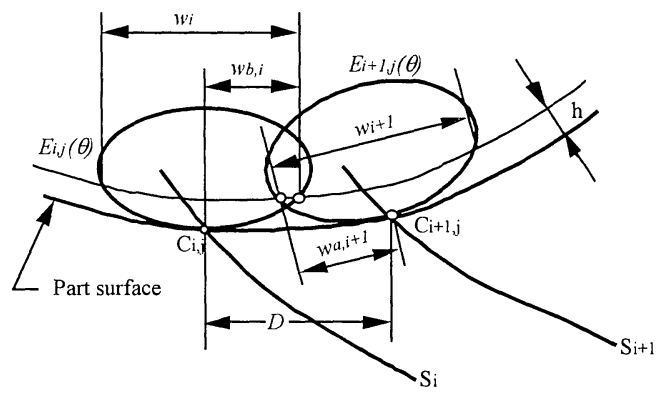

Si: Current tool path

$\mathrm{Si+1}$ : Adjacent new tool path

$\mathrm{C}_{\mathrm{i}, \mathrm{j}}$ : Cutter contact point on tool path $\mathrm{S}_{\mathrm{i}}$

$\mathrm{C} i+1, j$ : Adjacent cutter contact point on tool path $\mathrm{Si+1}$

For overlapping of machining strip: $w_{b, i}+w_{a, i+l}>D$

Figure 3. Overlapping of machining strips on adjacent tool paths

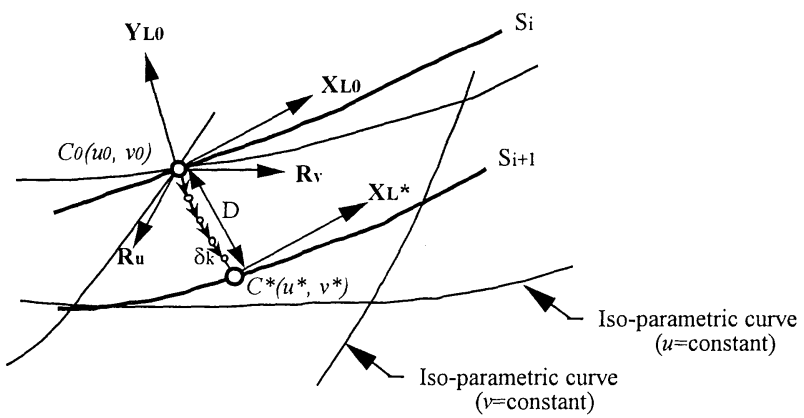

$\mathrm{Si}, \mathrm{Si}+1:$ Adjacent tool paths

$\mathrm{C} 0\left(u_{0}, v_{0}\right)$ : Current cutter contact point on tool path $\mathrm{S}_{\mathrm{i}}$

$\mathrm{C}^{*}\left(u^{*}, v^{*}\right)$ : Adjacent cutter contact point on tool path $\mathrm{S} i+1$

$\mathbf{R u}, \mathbf{R}$ : Tangential vectors at $\mathrm{C} 0$ along $u$ - and $v$-isoparametric directions

$\delta \mathrm{k}$ : Searching step size

$\mathbf{X} \mathbf{L}, \mathbf{X L}_{L^{*}}$ : Cutting directions at $\mathrm{C}_{0}$ and $\mathrm{C}^{*}$

$D$ : Path interval orthogonal to cutting direction $\mathbf{X}_{\mathbf{L}}$

Figure 4. Searching for the new adjacent cutter contact point $C^{*}\left(u^{*}, v^{*}\right)$ on path $S_{i+1}$

To calculate the precise tool path interval $D$, the direction of the path interval has to be orthogonal to the tool path itself [Lee 98a]. The orthogonal path interval cannot be adopted directly in parametric domains $(u, v)$ because the direction of the path interval is usually not oriented in the same direction as the constant parametric curve, as shown in Figure 4. Therefore, to find the optimal tool path distribution, we must solve the tool path interval $D$ and the parametric interval $(\Delta u$ or $\Delta v)$ in the orthogonal direction. As shown in Figure 4, a searching process is needed so that the feasible orthogonal path interval $D$ is equivalent to the 
feasible machining strip width as being constrained by Equation (2). The searching starts from the current cutter contact point $C_{0}$ and moves along the direction orthogonal to the cutting direction $\overrightarrow{\mathbf{X}_{\mathbf{L}}}$ to find the adjacent cutter contact point $C^{*}$. To maintain the accuracy, we calculate a searching step size $\delta_{k}$ based on the local surface curvature $\kappa$ and the predefined searching tolerance $\tau$, where $\tau$ is a sufficiently small tolerance. Given the current cutter contact point $C_{0}\left(u_{0}, v_{0}\right)$, the local surface curvature $\kappa$ along the orthogonal direction $\overrightarrow{\mathbf{Z}_{\mathbf{L}}}$-axis can be found. From Figure 5, one has the following relationship:

$$
\tau=\frac{1}{\kappa} ?(1-\cos \psi)
$$

The feasible step size $\quad k$ can be calculated as follows (also in Figure 5):

$$
\delta_{k}=2 ? \frac{1}{\kappa} ? \sin \psi
$$

where $\psi=\cos ^{-1}(1-\tau ? \kappa)$ from Equation (3). A conversion from the physical domain of step forward size $\left(\delta_{k} \overrightarrow{\mathbf{Z}_{\mathbf{L}}}\right)$ to the parameter increments $\left(\Delta u_{k}, \Delta v_{k}\right)$ in the parametric domain is needed in order to place the tool on the calculated adjacent path.

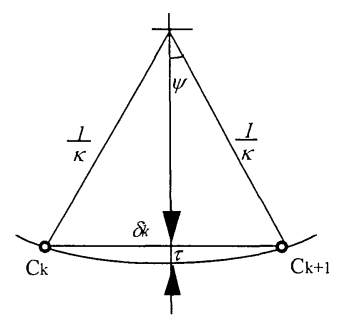

$\delta k$ : Feasible searching step size

$\tau$ : Step size tolerance

Figure 5. Determination of searching step size $\delta_{k}$ along path interval

\section{SOLVING PARAMETER INCREMENTS OF THE SEARCHING STEPS}

The parameter increments $\left(\Delta u_{k}, \Delta v_{k}\right)$ of a searching step $k$ along the orthogonal path interval direction can be found by using geometric analysis of the local area. As shown in Figure 6 , the searching vector $\vec{Z}_{\mathbf{L}}$ is defined by the current cutting direction $\vec{X}_{\mathbf{L}}$ and the surface normal $\overrightarrow{\mathbf{Y}_{\mathrm{L}}}, \quad\left(\overrightarrow{\mathbf{Z}_{\mathbf{L}}}=\overrightarrow{\mathbf{X}_{\mathbf{L}}} \times \overrightarrow{\mathbf{Y}_{\mathbf{L}}}\right)$. On the parametric part surface $R(u, v)$, the adjacent cutter contact point $C^{*}$ can be found by searching a series of marching points with a step size $\delta_{k}$ along the searching direction $\overrightarrow{\mathbf{Z}_{\mathbf{L}}}$ from the current cutter contact point $C_{0}\left(u_{0}, v_{0}\right)$, as shown in Figure 6(a). Figure 6(b) shows that, when moving along the searching direction $\vec{Z}_{\mathrm{L}}$ with a step size $\delta_{k}$, it is equivalent to move $\left(\Delta u \mathbf{R}_{\mathbf{u}}\right)$ along $\mathbf{R}_{\mathbf{u}}$ direction and $\left(\Delta v \mathbf{R}_{\mathbf{V}}\right)$ along $\mathbf{R}_{\mathbf{V}}$ direction, where $\mathbf{R}_{\mathbf{u}}$ and $\mathbf{R}_{\mathbf{V}}$ are the tangential vectors along $u$ - and $v$-parametric direction respectively. The parameter increments $\Delta u_{k}$ and $\Delta v_{k}$ in the parameter domain can be determined by solving the following two linear equations (see Figure 6):

$$
\begin{aligned}
& \Delta u_{k}\left(\mathbf{R}_{\mathbf{u}} ? \overrightarrow{\mathbf{X}_{\mathbf{L}}}\right)+\Delta v_{k}\left(\mathbf{R}_{\mathbf{v}} ? \overrightarrow{\mathbf{X}_{\mathbf{L}}}\right)=0 \\
& \Delta u_{k}\left(\mathbf{R}_{\mathbf{u}} ? \overrightarrow{\mathbf{Z}_{\mathbf{L}}}\right)+\Delta v_{k}\left(\mathbf{R}_{\mathbf{v}} ? \overrightarrow{\mathbf{Z}_{\mathbf{L}}}\right)=\delta_{k}
\end{aligned}
$$


where, $\overrightarrow{\mathbf{X}_{\mathbf{L}}}$ is the current cutting direction, $\overrightarrow{\mathbf{Z}_{\mathrm{L}}}$ is the searching direction (orthogonal to $\overrightarrow{\mathbf{X}_{\mathrm{L}}}$ ), $\mathbf{R}_{u}$ and $\mathbf{R}_{v}$ are the tangential vectors along $u$ and $v$, respectively. By solving Equations (5) and (6), we can find the parameter increments $\Delta u_{k}$ and $\Delta v_{k}$ as follows:

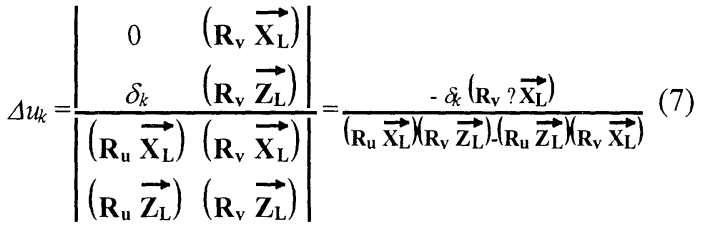

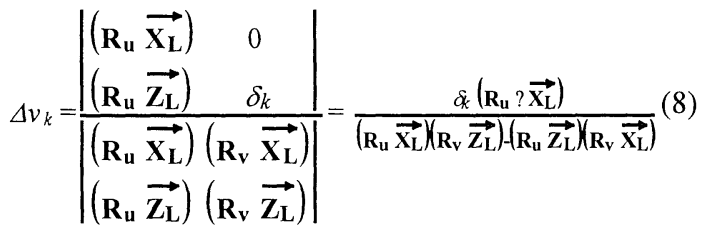

Let $\left(u_{k}, v_{k}\right)$ denote the current marching point $C_{k}$, then the new marching point $C_{k+1}\left(u_{k+1}\right.$, $v_{k+1}$ ) for the next searching can be found as follows:

$$
u_{k+1}=u_{k}+\Delta u_{k} \quad \text { and } \quad v_{k+1}=v_{k}+\Delta v_{k}
$$

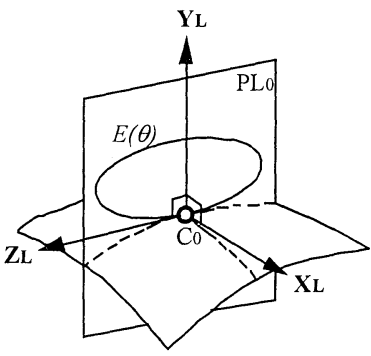

$\mathrm{C} 0$ : Current cutter contact point

$\mathrm{X}_{\mathbf{L}}$ : Cutting direction

YL: Surface normal

$Z_{L}$ : Path interval direction

$E(\theta)$ : Effective cutting shape on YL-ZL plane

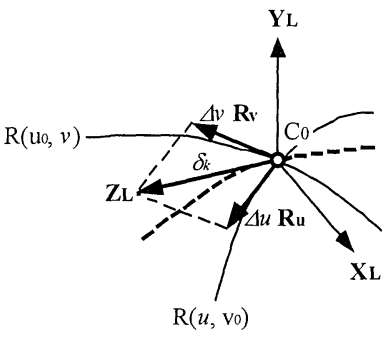

$\mathrm{C}_{0}$ : Current cutter contact point at $\mathrm{R}\left(\mathrm{u}_{0}, \mathrm{v}_{0}\right)$ $\mathrm{R}\left(u, v_{0}\right)$ : Iso-parametric curve along $v=v_{0}$ $\mathrm{R}\left(\mathrm{u}_{0}, v\right)$ : Iso-parametric curve along $u=\mathrm{u}_{0}$ $\delta$ : Step size along path interval direction $\mathrm{Z}_{\mathrm{L}}$

$\mathbf{R u}_{\mathbf{u}} \mathbf{R} \mathbf{s}$ : Tangential vectors along $u$ - and $v$ parametric curves

(b)

Figure 6. Solving parameter increments $u$ and $v$ of path interval along $\mathbf{Z}_{\mathbf{L}}$ axis

\section{FINDING THE ADJACENT CUTTER LOCATIONS}

Given a cutter contact point $C_{0}\left(u_{0}, v_{0}\right)$ on tool path $S_{i}$, we would like to find the adjacent cutter contact point $C^{*}$ on the new path $S_{i+1}$. The searching starts by finding an initial guess point $C_{g}$ on the adjacent tool path $S_{i+1}$. As shown in Figure 7, the machining strip width $w_{C O}$ at the current cutter contact point $C_{0}$ is calculated by offsetting a given tolerance $h$ from part surface. Assume $D$ is the path interval between the current cutter contact point $C_{0}$ and the guess point $C_{g}$, one can find the following relationship from Figure 7 as: 


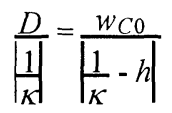

where, is the surface curvature normal to cutting direction $\overrightarrow{\mathbf{X}_{\mathbf{L}}}$. The distance $D$ between $C_{0}$ and $C_{g}$ can be represented as:

$$
D=\frac{w_{C 0}}{1-\kappa h}
$$

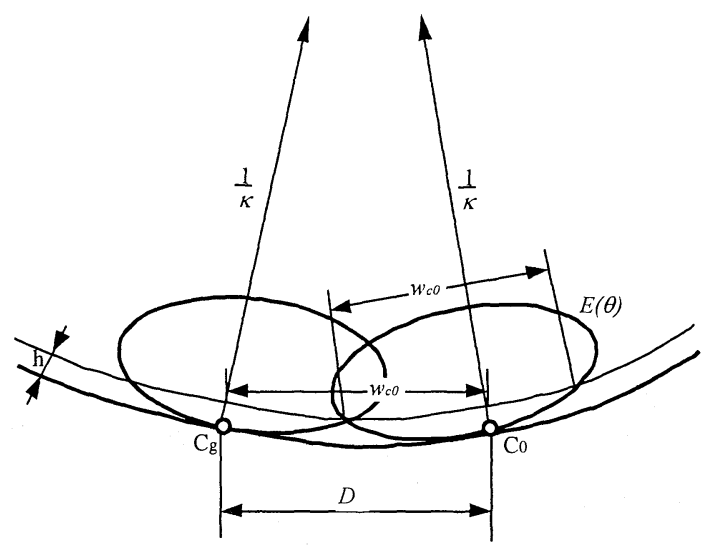

$\mathrm{C} 0$ : Current cutter contact point (on path $\mathrm{Si}$ )

$\mathrm{Cg}$ : Initial guess point for new cutter contact point on adjacent path $\mathrm{Si+1}$

Figure 7 Determination of the initial guess point $\mathrm{C}_{\mathrm{g}}$ using $w_{C} 0$ for adjacent tool path $\mathrm{S}_{\mathrm{i}+1}$

To find the initial guess point $C_{g}$, with a searching step size $\delta_{k}$ calculated by Equation (4), one needs to know the number of searching steps from $C_{0}$ to $C_{g}$. The number of searching steps, $n_{S}$, can be found by the following procedure as shown in Figure 8 . For each searching step of the marching point $C_{k}$ with step size $\delta_{k}$, one can find the following relationship from Figure 8:

$$
\psi_{k}=2 \sin ^{-1}\left(\frac{\delta_{k}}{2 \mid \frac{1}{k}}\right)
$$

For the distance $D$ calculated by Equation (11), we have the following relationship (Figure 8):

$$
\psi_{\text {Total }}=2 \sin ^{-1}\left(\frac{D}{2} \frac{1}{K}\right)
$$

From Figure 8, the following relationship exists:

$$
\psi_{\text {Total }}=n_{s} \psi_{k}
$$

Substituting Equations (12) and (13) into Equation (14), we can solve the number of searching steps, $n_{S}$, as follows:

$$
n_{s}=\frac{\sin ^{-1}\left(\frac{|k|}{2}\right)}{\sin ^{-1}\left(\frac{|k|}{2} \delta_{k}\right.}
$$


After marching along $\overrightarrow{\mathbf{Z}_{\mathbf{L}}}$ direction with $n_{S}$ searching steps, we can find the guess point $C_{g}\left(u_{g}\right.$, $v_{g}$ ) as follows (in Figure 8):

$$
C_{g}=\mathrm{R}\left(u_{g}, v_{g}\right)=\mathrm{R}\left(u_{0}+\sum_{k=1}^{n_{s}} \Delta u_{k}, v_{0}+\sum_{k=1}^{n_{s}} \Delta v_{k}\right)
$$

Using Equation (16), the machining strip width ${ }^{w} C_{g}$ at the cutter contact point $C_{g}$ can then be calculated. If the machining strip width between $C_{0}$ and $C_{g}$ is not equivalent to the path interval, i.e., $\left(w_{b, C g}+w_{a, C 0}<D-\varepsilon\right)$ or $\left(w_{b, C g}+w_{a, C 0}>D+\varepsilon\right)$ and $\varepsilon$ is a predefined searching tolerance, then the guess point $C_{g}$ is adjusted to find the new guess point $C_{g}$ '. The searching process is repeated until a satisfiable new guess point $C_{g}$ is found. Finally, the estimated guess point $C_{g}\left(u_{g}, v_{g}\right)$ is refined to obtain a "true" adjacent cutter contact point $C^{*}\left(u^{*}, v^{*}\right)$. For each cutter contact point $C_{i, j}$ of the current tool path $S_{i}$, the feasible path interval $w_{i, j}$ and the adjacent new cutter contact point $C_{i+1, j}$ on the next tool path $S_{i+l}$ can be calculated using the proposed non-isoparametric offset method, as shown in Figure 9.

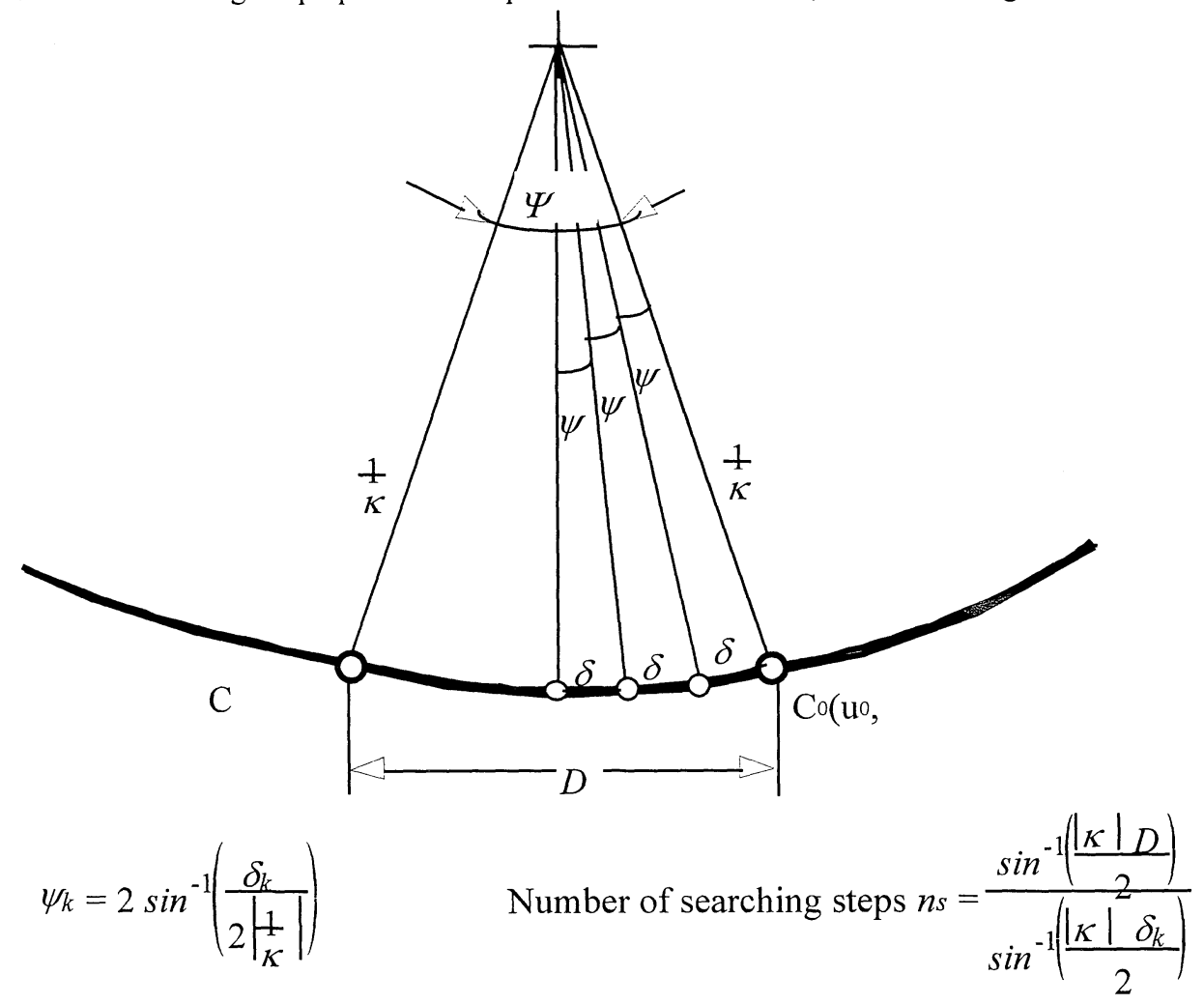

$\psi_{\text {Total }}=2 \sin ^{-1}\left(\frac{D}{2 \frac{1}{K}}\right) \quad$ Cutting direction

Figure 8. Determination of number $n_{S}$ of searching steps

As shown in Figure 10, if a non-isoparametric offset point $C^{\prime}$ that has at least one parameter value exceeds the parameter limits (either $u$ or $v$ parameter), then $C^{\prime}$ is skipped without being 
stored as an element of the new path $S_{i+1}$. If the error $f$ between any two cutter contact points, $\left(C_{i+1, j}, C_{i+1, j+1}\right)$ in sequence, is larger than the given tolerance, a new cutter contact point $C_{j}{ }^{\prime}$ is inserted between $C_{i+1, j}$ and $C_{i+1, j+1} . \quad C_{j}{ }^{\prime}$ can be defined as follows:

$$
C_{j}^{\prime}=R\left(\frac{\left(u_{i+1, j}+u_{i+1, j+1}\right)}{2}, \frac{\left(v_{i+1, j}+v_{i+1, j+1}\right)}{2}\right)
$$

where $R(u, v)$ is the surface description.

\section{COMPUTER IMPLEMENTATION AND RESULTS}

The methodology presented in this paper has been implemented using $\mathrm{C}$ programming language on Motorola PowerPC DT604 workstations. Some testing examples were reported in [Lee 98a]. For the testing example, the total tool path length generated by the traditional constant parametric method is 106.73 units. For the same example, the total length of the tool path generated by the proposed non-isoparametric method is 82.89 units. Comparing the result generated by the proposed non-isoparametric method and the one by the traditional method, one can easily find that the proposed method reduces the total tool path length of the example sculptured surface by $22.3 \%$. The reduction of total tool length by using the proposed non-isoparametric method should increase when the sculptured surface becomes more complex, due to the difference between the largest and smallest feasible machining strips along the constant parametric path. Details of the computer implementation and the testing results can be found in [Lee 98a].

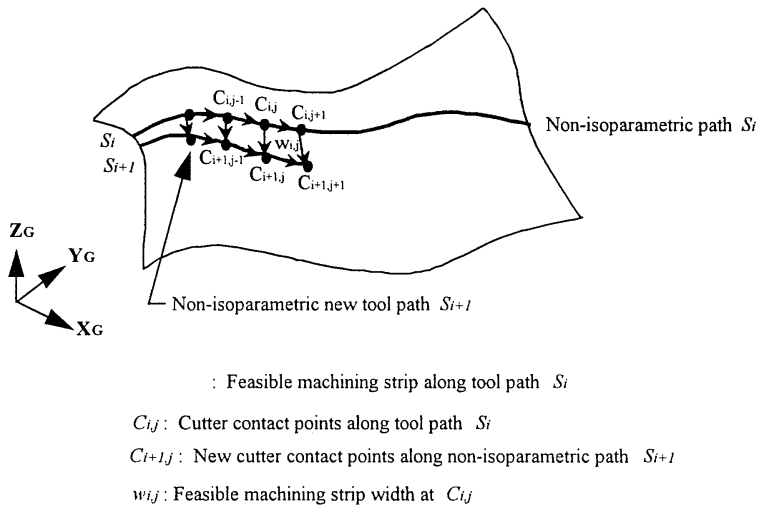

Figure 9 Finding the adjacent non-isoparametric tool path $S_{i+1}$ based on the calculated feasible path intervals

\section{CONCLUSIONS}

This paper presents a new approach to generating non-isoparametric tool paths for 5-axis sculptured surface machining. Two major contributions are explained in this paper. First, this paper deploys the new concept of machining strip analysis in non-isoparametric 5-axis tool path planning. Second, this paper presents a method that successfully searches for the parameter increments $(u, v)$ along an orthogonal path interval that enables a non-isoparametric path planning strategy to come true. The proposed method can be used to improve the 5-axis machining efficiency for $\mathrm{CAD} / \mathrm{CAM}$ systems. 


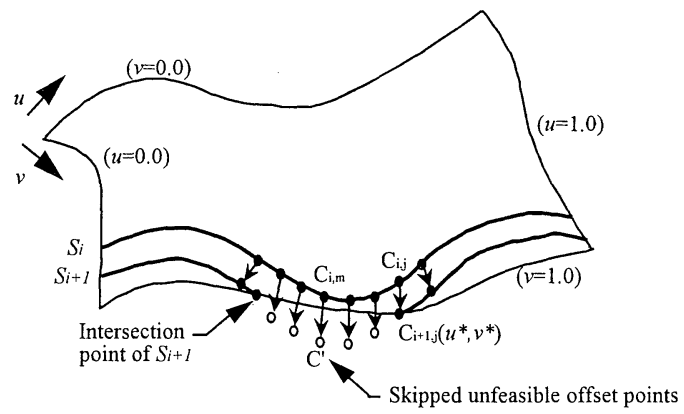

- : $\mathrm{C} i+1, \mathrm{j}$ feasible new cutter contact point on path $S_{i+1}$ $0:$ (Skipped) Infeasible offset point due to $\left(u^{*}>1.0\right)$ or
$\left(v^{*}>1.0\right)$ or $\left(u^{*}<0.0\right)$ or $\left(v^{*}<0.0\right)$

Figure 10. Skipping the unfeasible non-isoparametric offset points for path generation

\section{ACKNOWLEDGMENT}

This work was partially supported by the National Science Foundation (NSF) Faculty Early CAREER Award (DMI-9702374) to Dr. Y.S. Lee. Their support is greatly appreciated.

\section{REFERENCES}

1. Altan, T., et. al., "Advanced techniques for Die and Mold Manufacturing," Annals of CIRP, Vol.42, No.2, 1993, pp. 707-716.

2. Chen, Y.H., Lee, Y.S., and Fang, S.C., "Optimal Cutter Selection and Machining Plane Determination for Process Planning and NC Machining of Complex Surfaces," Journal of Manufacturing Systems, Vol. 17, No. 5, 1998, pp. 371-388.

3. Deng, Z., Leu, M.C., Wang, L., and Blackmore, D., "Determination of Flat-end Cutter Orientation in 5-Axis Machining," ASME International Mechanical Engineering Congress and Exposition, Atlanta, GA, November 1996, MED-Vol.4, pp. 73-80.

4. Jerard, R.B., Angleton, J.M., and Drysdale, R.L., "Sculptured Surface Tool Path Generation with Global Interference Checking," ASME Design Productivity International Conference, Honolulu, Hawaii, February 1991, pp. 737-742.

5. Lee, Y.S., and Chang, T.C., "Application of Computational Geometry in Optimizing 2.5 D and 3D NC Surface Machining," Computers in Industry, Vol. 26, No. 1, 1995a, pp. 41-59.

6. Lee, Y.S., and Chang, T.C., "Two-Phase Approach to Global Tool Interference Avoidance in 5-axis Machining", Computer Aided Design, Vol. 27, No. 10, 1995b, pp. 715-729.

7. Lee, Y.S., and Chang, T.C., "Machined Surface Error Analysis For 5-axis Machining," International Journal of Production Research, Vol. 34, No. 4, 1996a, pp. 977-998.

8. Lee, Y.S., and Chang, T.C., "Automatic Cutter Selection for 5-axis Sculptured Surface Machining," the International Journal of Production Research, Vol. 34, No. 4, 1996b, pp. 977-998.

9. Lee, Y.S. and Ji, H, "Surface Interrogation and Machining Strip Evaluation for Multi-axis CNC Die/Mold Machining," International Journal of Production Research, Vol. 35, No. 1, 1997a, pp. 225-252.

10. Lee, Y.S., "Admissible Tool Orientation Control of Gouging Avoidance for 5-Axis Complex Surface Machining," Computer-Aided Design, Vol. 29, No. 7, 1997b, pp. 507-521.

11. Lee, Y.S., "Non-isoparametric Tool Path Planning by Machining Strip Evaluation for 5-Axis Sculptured Surface Machining," Computer-Aided Design, Vol. 30, No. 7, 1998a, pp. 559-570.

12. Lee, Y.S., "Mathematical Modeling of Using Different Endmills and Tool Placement Problems For 4and 5-Axis NC Complex Surface Machining," International Journal of Production Research, Vol. 36, No. 3, 1998b, pp. 785-814.

13. Li, S., and Jerard, R.B., "5-axis Machining of Sculptured Surfaces with a Flat-end Cutter," Computer Aided Design, Vol. 26, No. 3, 1994, pp. 165-178. 
14. Lin, R.S., and Koren, Y., "Efficient Tool-path Planning for Machining Free-form Surfaces," Transactions of the ASME, Vol. 118, No. 1, 1996, pp. 20-28.

15. Ma, Y., and Lee, Y.S., "Detection of Loops and Singularities of Surface Intersections," (in press) Computer-Aided Design, Accepted on September 22, 1998.

16. Suresh, K., and Yang, D.C.H., "Constant Scallop Height Machining of Free-form Surfaces," ASME Journal of Engineering for Industry, Vol. 116, No. 2, 1994, pp. 253-259.

17. Vickers, G.W., and Quan, K.W., "Ball-mills versus end-mills for curved surface machining", Journal of Engineering for Industry, Vol.111, February, 1989, pp. 22-26. 